\title{
Biossegurança na odontologia
}

\section{Biosafety in dentistry}

André Luiz Dantas Bezerra', Milena Nunes Alves de Sousa², Ankilma do Nascimento Andrade Feitosa ${ }^{3}$, Elisangela Vilar de Assis ${ }^{3}$, Criseuda Maria Benício Barros ${ }^{1}$, Eclivaneide Caldas de Abreu Carolino ${ }^{3}$

'Universidade Estadual da Paraíba (UEPB) - Campina Grande (PB), Brasil.

${ }^{2}$ Universidade de Franca (UNIFRAN) - Franca (SP), Brasil.

${ }^{3}$ Faculdade Santa Maria (FSM) - Cajazeiras, (PB), Brasil.

DOI: http://dx.doi.org/10.7322/abcshs.v39i1.251

\section{RESUMO}

Introdução: As atividades laborais, de modo geral, expõem os trabalhadores a riscos ocupacionais dos mais variados. Esses riscos incluem agentes físicos, químicos, ergonômicos, de acidentes e biológicos. O contato rotineiro com esses últimos deve ser motivo de reflexão e intervenção. Objetivo: Descrever a produção científica sobre a biossegurança na odontologia. Métodos: Foi realizada uma revisão do estado da arte, adotando-se critérios para análise dos trabalhos mais diretamente relacionados à prática odontológica. A partir dos descritores controlados (DeCS) "biossegurança" e "odontologia", procederam-seabuscanaLiteratura Latino-Americana e do Caribe em Ciências da Saúde, na Scientific Electronic Library Online, na National Library of Medicine e na Biblioteca Cochrane. Resultados: Com a associação entre os DeCS, chegou-se a 66 artigos, porém, após refinamento foram incluídos nesta revisão 12 artigos. Nos resultados foi evidenciado que a produção científica sobre biossegurança é vasta, bem como é ampla na odontologia, contudo, quando da concentração de trabalhos contempladores da associação dos termos biossegurança e odontologia, a quantidade de estudos diminui consideravelmente. Não obstante, as pesquisas foram, em sua maioria, desenvolvidas por estudiosos nacionais, da região nordeste e evidenciaram que o conhecimento e a adoção de medidas de segurança biológica na área ainda são insuficientes para garantir a proteção da equipe e dos pacientes qualitativamente. Conclusão: Sugere-se que as normas de precaução padrão sejam seguidas rigidamente e que ações de educação sejam promovidas entre os profissionais.

Palavras-chave: fatores biológicos; exposição a agentes biológicos; odontologia.

\section{ABSTRACT}

Introduction: Work activities in dentistry generally expose workers to a variety of occupational hazards. This hazards include physical and chemical agents, ergonomic, biological and accident risks. The routine contact with them should be a cause for reflection and intervention. Objective: To describe the scientific literature on biosafety in dentistry. Methods: The authors conducted a review of the state of the art, adopting criteria for evaluating the works most directly related to dental practice. From the controlled descriptors (MeSH) "biosafety" and "dentistry", a search was conducted in the Latin American and Caribbean Health Sciences, the Scientific Electronic Library Online, the National Library of Medicine and the Cochrane Library databases. Results: Based on the association between keywords, 66 items were found. Aafter refinement, 12 articles were included in this review. Results showed that the scientific literature on biosafety is vast and broad, as well as in dentistry. However, when the association of biosafety and dentistry keywords is made, the number of studies decreases considerably. Nevertheless, the surveys were mostly developed by national experts from the Northeast region of Brazil and showed that the awareness and adoption of biosecurity measures in the area is still insufficient to ensure the protection of staff and patients qualitatively. Conclusion: It is suggested that the rules of standard precautions be rigidly followed, and that education activities be promoted among professionals.

Keywords: biological factors; exposure to biological agents; dentistry.

Recebido em: 14/01/2012

Revisado em: 10/06/2012

Aprovado em: 16/06/2012 


\section{INTRODUÇÃO}

As atividades laborais, de modo geral, expõem os trabalhadores a riscos ocupacionais dos mais variados. Esses riscos envolvem agentes físicos, químicos, ergonômicos, de acidentes e biológicos. O contato rotineiro com esses últimos deve ser motivo de reflexão e intervenção. Deste modo, o controle das doenças infecciosas tem sido constante e uma ascendente preocupação entre os profissionais de saúde, pois estes e os pacientes se tornam disseminadores de doenças.

Essa inquietação também deve ser corrente na área odontológica. Segundo Krieger et al. ${ }^{1}$, os trabalhadores da odontologia estão cotidianamente sujeitos a inúmeras formas de contaminação por agentes biológicos patogênicos, os quais se apresentam em fluídos orgânicos, como sangue e saliva, que são manuseados comumente por tais profissionais. Esses agentes são capazes de causar enfermidades infectocontagiosas graves e ainda possibilitar a ocorrência de um ciclo de infecção cruzada dentro e fora do ambiente odontológico, tornando-se indispensável adotar medidas que minimizem os efeitos que os mesmos podem ocasionar.

Apesar de haver medidas de precaução padrão, as quais mantêm seguros o profissional e o usuário do serviço, o desconhecimento, a utilização errônea de métodos de esterilização, a resistência bacteriológica e de diversos tipos de vírus e a ausência de cuidado dos profissionais com situações de risco têm colaborado para a elevação do número de casos de infecções por vírus, especialmente pelo vírus da hepatite $\mathrm{B}(\mathrm{HBV})$ e o vírus da hepatite $\mathrm{C}(\mathrm{HCV})$, em profissionais e pacientes, adquiridas por meio dos procedimentos diversos, tanto na área médica como na odontológica.

Diante dos elementos discorridos, bem como do risco aumentado de desenvolvimento de doenças ocupacionais de cunho biológico e de acidentes, o que torna os cirurgiões-dentistas um dos profissionais mais suscetíveis, se despertou pela temática e houve o interesse em realizar esta pesquisa.

Assim, o objetivo é descrever a produção científica sobre a biossegurança na odontologia.

\section{MÉTODO}

A realização desta revisão integrativa da literatura foi realizada entre os meses de abril e junho de 2012, com determinação da temática e formulação de uma pergunta-problema: qual o perfil das publicações sobre biossegurança na odontologia? Na sequência, buscou-se estabelecer os critérios de inclusão e exclusão, os quais possibilitaram contemplar pesquisas referentes ao fenômeno estudado.

Deste modo, foram estabelecidos pelos autores os seguintes critérios de inclusão: publicações dos últimos oito anos (2005 a 2012); trabalhos disponíveis na íntegra em formato de artigo científico, os quais apresentassem pelo menos um dos descritores (biossegurança e odontologia) no título; e de acesso gratuito. Em contrapartida, foram desconsideradas obras anteriores a 2005; publicações repetidas; produções não relacionadas à temática; artigos cujo texto completo estava indisponível gratuitamente; obras que não apresentaram ao menos um dos descritores selecionados no título; além de leis, capítulos de livros, monografias, dissertações e teses.

Posteriormente, foi realizada a identificação dos estudos pré-selecionados e selecionados. Para tal, foi utilizada a Biblioteca Virtual de Saúde (BVS), considerada uma base de dados confiável. Para busca dos artigos na Literatura Latino-Americana e do Caribe em Ciências da Saúde (LILACS), na Scientific Electronic Library Online (SciELO), na National Library of Medicine (MEDLINE) e na Biblioteca Cochrane foram utilizados os descritores Controlados em Ciências da Saúde (DeCS) "biossegurança" e "odontologia". Inicialmente, tais descritores foram analisados de forma isolada, obtendo-se diversas produções.

Com o refinamento dos artigos foram selecionados 66 artigos potencias. Neste momento, foi feita a leitura dos resumos, palavras-chave e título das publicações, organizando os estudos pré-selecionados e identificando os selecionados.

A identificação das pesquisas, a partir dos critérios de inclusão, possibilitou chegar a 12 artigos. De posse desses, buscou-se extrair as informações úteis para a revisão, as quais contemplassem o objetivo da mesma. Nessa fase, foi adotado como instrumento de coleta a matriz de síntese ou de análise.

\section{RESULTADOS E DISCUSSÃO}

Salienta-se que produção científica sobre biossegurança é vasta, bem como também é ampla na odontologia, porém, quando da concentração de trabalhos contempladores da associação entre esses dois DeCS, a quantidade de estudos diminui consideravelmente, como verificado nesta revisão integrativa da literatura. Assim sendo, após criterioso refinamento e análise, considerando os critérios de inclusão delineados, chegou-se a 12 artigos, dos quais nove $(75,0 \%)$ estavam disponíveis na LILACS, dois $(16,7 \%)$ na SciELO e um $(8,3 \%)$ na MEDLINE.

Em relação ao idioma de publicação, a maioria foi publicado em língua portuguesa $(83,4 \% ; n=10)$. Um artigo $(8,3 \%)$ foi publicado em inglês e outro $(8,3 \%)$ em espanhol. O dado pode estar relacionado à base de dados utilizados para pesquisa, pois se utilizou a BVS, uma biblioteca digital nacional.

Em relação ao ano de publicação, nota-se que entre os períodos incluídos nesta revisão (2005 a 2012), houve maior publicação em $2009(41,7 \% ; n=5)$ e em $2010(16,7 \% ; n=2)$. Os demais anos foram representados por $8,3 \%(\mathrm{n}=1)$ cada. Ressalta-se, entretanto, que não houve publicação sobre a temática em 2012.

Aconcentraçãononúmerodepublicaçõessobreoobjetodeestudo neste período pode ser justificada pelo advento da NR 32, publicada pelo Ministério do Trabalho e Emprego na Portaria do Gabinete Ministerial (GM) no 485, de novembro de $2005^{2}$ e (re)publicada na portaria GM no 939 , de novembro de $2008^{3}$ e também na Portaria GM n ${ }^{\circ} 1.748$, de agosto de $2011^{4}$. Nessas legislações infraconstitucionais é estabelecida a obrigatoriedade da segurança e saúde no trabalho nos serviços de saúde, o que contempla as clínicas odontológicas. 
A NR 32, entre tantos aspectos, induz a necessidade premente das instituições de saúde adotarem as medidas de precaução padrão, estabelecendo as diretrizes básicas para a implementação de medidas de proteção à segurança e à saúde dos trabalhadores dos serviços de saúde, bem como aqueles que exercem atividades de promoção e assistência à saúde em geral ${ }^{2-4}$.

Em relação aos tipos de pesquisa, verificou-se que a maioria não especificou a tipologia adotada $(33,3 \% ; n=5)$, seguindo-se pelos estudos de caráter qualitativo $(19,3 \% ; \mathrm{n}=3)$ e observacionais $(13,3 \% ; \mathrm{n}=2)$; e um $(6,7 \%)$ de campo, descritivo, experimental e exploratório, cada.

Quanto à população do estudo, a grande maioria abordou os estudantes de odontologia $(66,7 \% ; n=9)$. Por conseguinte, $16,7 \%(n=2)$ pesquisaram os cirurgiões-dentistas e $8,3 \%(n=1)$ a literatura (estudos de revisão). Tais resultados fazem emergir as seguintes questões/inquietações: somente os estudantes são passíveis de erros quanto à biossegurança na odontologia? Os profissionais sempre utilizam as normas?

A partir dessas perguntas, pode-se afirmar que a inexperiência dos estudantes de odontologia durante os procedimentos, ao contrário daquele profissional com mais prática, é capaz de induzir o respeito às normas de biossegurança, já que o medo de adquirir uma doença infectocontagiosa ainda é frequente. O cirurgião-dentista, confiando em sua experiência tende a negligenciá-las.

Jorge $^{5}$ e Engelmann et al. ${ }^{6}$ concordam com a assertiva quando afirmam que o profissional da odontologia é negligente em implantar os métodos descritos na literatura como eficazes na manutenção da cadeia asséptica.

Em relação à instituição de filiação dos autores, nota-se uma grande diversidade, incluindo universidades estaduais, federais e privadas, bem como de regiões diferentes e estrangeiras. Destaque para a Universidade Federal de Pernambuco (UFPE) e Universidade Estadual Paulista (UNESP), com 16,8\% ( $\mathrm{n}=2$ ) cada. As demais instituições de ensino superior foram representadas por $8,3 \%(\mathrm{n}=1)$, cada.

Ainda, a região nordeste mostrou-se como pólo científico no que concerne à publicação sobre a temática, visto que foi representada por $50,0 \%(n=6)$ das publicações, seguida pela região sudeste, com 25,1\% ( $\mathrm{n}=3)$. O centro-oeste, sul e os países estrangeiros só foram representados por $8,3 \%(\mathrm{n}=1)$, cada. $\mathrm{O}$ norte não obteve representação.

A pesquisa evidencia que os métodos de biossegurança usados pelos profissionais da odontologia, independentemente de sua região, estão sendo desobedecidos, culminando em possíveis disseminações de enfermidades. Apesar disso, os membros da equipe de saúde bucal reconhecem a necessidade de aplicar os métodos instituídos ao controle da infecção cruzada? .

Por conseguinte, a análise dos artigos incluídos nesta revisão integrativa foi iniciada com vistas a identificar o objeto de estudo das pesquisas desenvolvidas na atualidade sobre a biossegurança na odontologia. Fundamentando-se nos conteúdos dos artigos, quatro abordagens temáticas ou enfoques centrais surgiram: (1) utilização de precauções universais; (2) bioética na biossegurança; (3) conhecimento e importância atribuída à biossegurança; e (4) materiais utilizados para efetivação da biossegurança (Tabela 1).

A seguir estão expostas as principais contribuições dos estudos catalogados nesta revisão, no período de 2005 a 2012. Ressaltase, antecipadamente, que a maioria das propostas $(50,0 \% ; n=6)$

Tabela 1: Distribuição dos artigos incluídos segundo abordagens temáticas

\begin{tabular}{|c|c|c|}
\hline Estudos & Utilização de precauções universais & n (\%) \\
\hline Garbin et al. ${ }^{8}$ & $\begin{array}{l}\text { Avaliar descritivamente a aplicação de medidas de precaução universal para controle de infecção entre } \\
\text { cirurgiões-dentistas que atuam em consultórios públicos e particulares do município de Araçatuba, São Paulo. }\end{array}$ & \multirow{6}{*}{$6(50,0)$} \\
\hline Pimentel et al. ${ }^{9}$ & Verificar o comportamento dos acadêmicos de odontologia no combate à infecção cruzada. & \\
\hline Orestes-Cardoso et al. ${ }^{10}$ & $\begin{array}{l}\text { Identificar a prevalência de acidentes perfurocortantes e as medidas profiláticas pós-acidentes em } \\
\text { duas Faculdades de Odontologia de Recife, Pernambuco, Brasil. }\end{array}$ & \\
\hline Vasconcelos et al. ${ }^{11}$ & $\begin{array}{l}\text { Observar o cumprimento das normas de biossegurança e os cuidados com os riscos ocupacionais } \\
\text { pelos alunos que atenderam nas clínicas de Odontologia Preventiva, Odontopediatria, Dentística, } \\
\text { Endodontia e nos procedimentos cirúrgicos nas clínicas de Periodontia e Cirurgia, da Faculdade de } \\
\text { Odontologia da Universidade Federal de Pernambuco (UFPE). }\end{array}$ & \\
\hline Oliveira et al. ${ }^{12}$ & $\begin{array}{l}\text { Avaliar as atitudes de prevenção de infecção através de inspeção visual nas clínicas de graduação da } \\
\text { Faculdade de Odontologia de Pernambuco (FOP/UPE). }\end{array}$ & \\
\hline Pinelli et al. ${ }^{13}$ & $\begin{array}{l}\text { Investigar as percepções de graduandos de odontologia sobre a fidelidade às diretrizes de } \\
\text { biossegurança e acerca do preservar-se. }\end{array}$ & \\
\hline Estudos & Bioética na biossegurança & n (\%) \\
\hline Mengarelli $i^{14}$ & Discutir a bioética como suporte para a biossegurança. & \multirow[b]{2}{*}{$2(16,7)$} \\
\hline Bugarin Júnior e Garrafa ${ }^{15}$ & $\begin{array}{l}\text { Analisar o uso de biomateriais em odontologia por cirurgiões dentistas especialistas sob os aspectos } \\
\text { da Teoria Bioética do Principialismo e da ética da responsabilidade individual e coletiva. }\end{array}$ & \\
\hline Estudos & Conhecimento e importância atribuída às normas de biossegurança & $\mathbf{n}$ \\
\hline Santos et al. ${ }^{16}$ & $\begin{array}{l}\text { Avaliar o conhecimento de acadêmicos do curso de odontologia sobre a desinfecção de moldes de } \\
\text { hidrocoloide irreversível. }\end{array}$ & \multirow{3}{*}{$3(25,0)$} \\
\hline Diniz et al. ${ }^{17}$ & $\begin{array}{l}\text { Observar o conhecimento de acadêmicos de odontologia para melhor definir diretrizes de } \\
\text { biossegurança em ambiente radiológico. }\end{array}$ & \\
\hline Schroeder et al. ${ }^{18}$ & $\begin{array}{l}\text { Avaliar o grau de importância da biossegurança na visão dos alunos do curso de graduação de } \\
\text { odontologia da Universidade da Região de Joinville, Santa Catarina. }\end{array}$ & \\
\hline Estudos & Materiais utilizados para efetivação da biossegurança & $\mathbf{n}$ \\
\hline Lopes et al. ${ }^{19}$ & $\begin{array}{l}\text { Analisar a permeabilidade das luvas de látex para procedimento mais usados por alunos da Faculdade } \\
\text { de Odontologia da Universidade Federal da Bahia (UFBA). }\end{array}$ & $1(8,3)$ \\
\hline
\end{tabular}


limitou-se à utilização de precauções padrões, pouco evidenciando as condutas para sua efetivação prática.

Para Garbin et al. ${ }^{8}$, o uso de gorro foi relatado por 55\% dos cirurgiões-dentistas que atuam no serviço público e $90 \%$ dos que atuam no serviço privado. O uso de máscara e o uso de luvas foram relatados pelos 40 profissionais entrevistados, no entanto, a troca de luvas entre os pacientes não foi relatada por $40 \%$ dos profissionais da rede pública. Já Mengarelli ${ }^{14}$ destaca que durante a atuação do profissional da odontologia no Chile não é obrigatória a adoção de medidas de biossegurança, deixando a critério de cada cirurgião-dentista a sua aplicabilidade. No estudo de Bugarin Júnior e Garrafa ${ }^{15}$, destaca-se que os profissionais utilizam biomateriais desconhecendo riscos e efeitos adversos, distanciando-se do princípio da beneficência.

Já nos relatos de Santos et al. ${ }^{16}, 90 \%$ dos indivíduos afirmaram saber que o molde de hidrocoloide irreversível pode disseminar doenças infectocontagiosas, mas apenas $66 \%$ responderam que aprenderam uma técnica de desinfecção do molde. Muitos acadêmicos (41\%) não souberam responder qual substância seria indicada para a desinfecção de moldes de hidrocoloide irreversível e apenas 70\% lavavam o molde em água corrente antes da desinfecção química. Por outro lado, Pimentel et al. ${ }^{9}$ relatam que dos entrevistados, 22,22\% utilizam sempre todos os equipamentos de proteção individual (EPIs), 70,9\% não evitam tocar superfícies quando enluvados; $85,2 \%$ lavam as mãos antes e depois do atendimento, $15,7 \%$ acreditam que luvas não tem microperfurações e todos utilizam sabão líquido e papel toalha.

Orestes-Cardoso et al. ${ }^{10}$ constataram que a prevalência de alunos acidentados nas duas instituições foi de $25,3 \%$, com percentual mais elevado nos alunos do $8^{\circ}$ ao $10^{\circ}$ períodos $(35,3 \%)$. Dos acidentados, $34,2 \%$ foram orientados por professores em relação às medidas profiláticas. A maioria $(73,7 \%)$ se restringiu a lavar o ferimento com água e sabão. Apenas 13,2\% procuraram serviço médico especializado em acidentes ocupacionais, no entanto, $88,7 \%$ classificaram o conhecimento que tinham acerca de medidas profiláticas pós-acidentes de razoável a bom. Já Lopes et al. ${ }^{19}$ relatam que luvas de látex para procedimento são eficazes como EPI, porém o tempo de uso deve ser observado criteriosamente, pois há a possibilidade de permeabilidade após o uso prolongado, podendo haver contaminação do usuário.

Para Vasconcelos et al. ${ }^{11}$, os resultados apontaram ausência de manilúvio, inadequações no uso dos EPIs para alunos e pacientes, falta de local exclusivo para lavagem de instrumental e deficiências ergonômicas durante a prática clínica. Noutra contribuição, Diniz et al. ${ }^{17}$ descrevem que foi observado que $90 \%$ dos acadêmicos se mostraram preocupados com a biossegurança, dos quais 55\% admitiram desconhecer o tipo de desinfetante utilizado para controle de infecção. Em relação à proteção radiológica, $94 \%$ dos acadêmicos estavam preocupados com os efeitos da radiação ionizante, $76 \%$ tinham conhecimento quanto à necessidade de modificar o tempo de exposição segundo a área e a idade do paciente, porém $59 \%$ não sabiam que filme utilizar.

Nos achados de Oliveira et al. ${ }^{12}$, o ambiente de trabalho das clínicas da Faculdade de Odontologia de Pernambuco apresentou falhas, não inviabilizando, contudo, a prática clínica. $\mathrm{O}$ controle de infecção cruzada por parte dos operadores foi inadequado. Para Schroeder et al..$^{18}$, a grande maioria dos estudantes demonstrou ter conhecimentos básicos sobre as normas de biossegurança (82,39\%) e a utilização de EPIs (75,35\%).

Já Pinelli et al. ${ }^{13}$ verificaram a adesão dos entrevistados aos protocolos de biossegurança, embora houvesse a queixa de que, na rotina diária, as precauções fossem negligenciadas por não serem muito práticas. Entre as medidas de proteção individual e coletiva rotineiramente utilizadas, foram apontados o uso de EPI e as barreiras protetoras, bem como as atividades de desinfecção e esterilização.

Assim, os estudos listados inferem-nos a descrever que a equipe de saúde bucal, bem como os usuários dos serviços odontológicos, estão expostos aos riscos biológicos de modo considerável, haja vista que as normas e condutas de biossegurança, em geral, estão sendo desrespeitadas ou respeitadas parcialmente. Ainda, há que se considerar que o profissional tem a obrigatoriedade de atuar fundamentado na ética/bioética, a qual preconiza, entre tantos aspectos, a segurança do paciente, mas este elemento também não tem sido respeitado.

Há um quadro de insuficiência na aplicabilidade e conhecimento das medidas de segurança biológica, embora apontem para a utilização de precauções universais, para o reconhecimento das normas a serem respeitadas pela equipe odontológica. No mais, trazem a escassez ou limitação de condutas de biossegurança, o uso de biomaterias sem conhecimento e sem respeito à bioética e os acidentes com material biológico.

Sugere-se que as normas de precaução padrão sejam seguidas rigidamente e que ações de educação sejam promovidas entre os profissionais.

\section{REFERÊNCIAS}

1. Krieger D, Bueno R, Gabardo MCL. Perspectivas de biossegurança em odontologia. Rev Gestão Saúde. 2010;1(2):1-10.

2. Brasil. Ministério do Trabalho e Emprego. Portaria GM $n^{\circ} 485$, de 11 de novembro de 2005. Dispõe sobre a segurança e saúde no trabalho em serviços de saúde. Brasília: Diário Oficial da União; 2005. Seção 1 .

3. Brasil. Ministério do Trabalho e Emprego. Portaria GM n 939, de 18 de novembro de 2008. Dispõe sobre a segurança e saúde no trabalho em serviços de saúde. Brasília: Diário Oficial da União; 2008.
4. Brasil. Ministério do Trabalho e Emprego. Portaria GM n 1.748, de 30 de agosto de 2011. Dispõe sobre a segurança e saúde no trabalho em serviços de saúde. Brasília: Diário Oficial da União; 2011.

5. Jorge AOC. Princípios de biossegurança em odontologia Rev Biociênc. 2002;8(1):7-17.

6. Engelmann Al, Daí AA, Miura CSN, Bremm LL, Boleta-Ceranto DCF Avaliação dos procedimentos realizados por cirurgiões-dentistas da região de Cascavel-PR visando ao controle da biossegurança. Odontol Clín-Cient. 2010;9(2):161-5 
7. Machado GL, Kather JM. Estudo do controle da infecção cruzada utilizada pelos cirurgiões-dentistas de Taubaté. Rev Biociênc. 2002;8(1):37-44.

8. Garbin AJ, Garbin CA, Arcieri RM, Crossato M, Ferreira NF. Biosecurity in public and private office. J Appl Oral Sci. 2005;13(2):163-6. http://dx.doi.org/10.1590/S1678-77572005000200013

9. Pimentel MJ, Batista Filho MMV, Rosa MRD, Santos JP. Utilização dos equipamentos de proteção individual pelos acadêmicos de Odontologia no controle da infecção cruzada. Rev Bras Odontol. 2009;66(2):211-5.

10. Orestes-Cardoso SM, Farias ABL, Pereira MRMG, OrestesCardoso AJ, Cunha Júnior IF. Acidentes perfurocortantes: prevalência e medidas profiláticas em alunos de odontologia. Rev Bras Saúde Ocup. 2009;34(119):6-14. http://dx.doi.org/10.1590/S0303-76572009000100002

11. Vasconcelos MMVB, Brasi CMV, Mota CCBO, Carvalho NR. Avaliação das normas de biossegurança nas clínicas odontológicas da UFPE. Odontologia Clín-Científ. 2009;8(2):151-6.

12. Oliveira GMF, Ribeiro GA, Oliveira PM, Burgos MEA. Avaliação das atitudes de prevenção de infecção cruzada através de inspeção visual nas clínicas de graduação da Faculdade de Odontologia de Pernambuco - FOP/UPE. Odontol Clín-Cient. 2010;9(4):349-53.

13. Pinelli C, Garcia PPNS, Campos JADB, Dotta AAV, Rabello AP. Biossegurança e odontologia: crenças e atitudes de graduandos sobre o controle da infecção cruzada. Saúde Soc. 2011;20(2):448-61. http://dx.doi.org/10.1590/S0104-12902011000200016

14. Mengarelli RRI. La bioética como soporte de la bioseguridad. Acta Bioeth. 2006;12(1):29-34

http://dx.doi.org/10.4067/S1726-569X2006000100004

15. Bugarin Júnior JG, Garrafa V. Bioética e biossegurança: uso de biomateriais na prática odontológica. Rev Saúde Pública. 2007;41(2):223-8.

http://dx.doi.org/10.1590/S0034-89102007000200008

16. Santos FS, Scannavino FLF, Martins AT, Oliveira SC, Dias AP, Rodrigues RV. Conhecimento de acadêmicos em Odontologia sobre a desinfecção de moldes de hidrocolóide irreversível. Rev Odonto Ciênc. 2008;23(4):371-4

17. Diniz DN, Bento PM, Pereira MSV, Pereira JV, Silva DF, Costa MRM, et al. Avaliação do conhecimento sobre biossegurança em radiologia pelos alunos do curso de Odontologia da Universidade Estadual da Paraíba. Arq Ciênc Saúde. 2009;16(4):166-9.

18. Schroeder MDS, Marin C, Miri F. Biossegurança: grau de importância na visão dos alunos do curso de graduação de Odontologia da Univille. Rev Sul-Bras Odontol. 2010;7(1):20-6.

19. Lopes N, Prates N, Rabelo R, Wanderley-Cruz JF. Análise da permeabilidade das luvas de látex para procedimento mais utilizadas por alunos da Faculdade de Odontologia da Universidade Federal da Bahia. R Ci Méd Biol. 2009;8(2):206-12. 